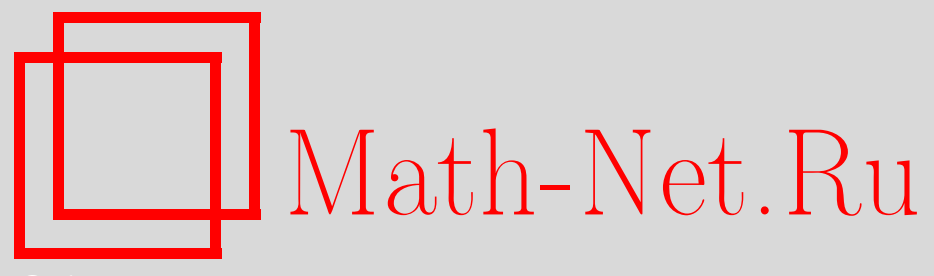

С. В. Сазонов, Н. В. Устинов, Интегрируемые модели динамики продольно-поперечных акустических импульсов в парамагнитном кристалле, ТМФ, 2007, том 151, номер 2, 228-247

DOI: https://doi.org/10.4213/tmf6042

Использование Общероссийского математического портала Math-Net.Ru подразумевает, что вы прочитали и согласны с пользовательским соглашением http://www.mathnet.ru/rus/agreement

Параметры загрузки:

IP : 18.207 .199 .55

26 апреля 2023 г., 15:51:39

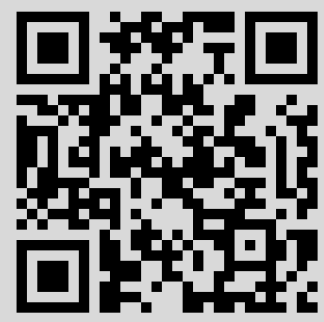




\title{
ИНТЕГРИРУЕМЫЕ МОДЕЛИ ДИНАМИКИ ПРОДОЛЬНО-ПОПЕРЕЧНЫХ АКУСТИЧЕСКИХ ИМПУЛЬСОВ В ПАРАМАГНИТНОМ КРИСТАЛЛЕ
}

\begin{abstract}
Изучено взаимодействие продольно-поперечных акустических импульсов с системой парамагнитных примесей с эффективным спином $S=1$, находящейся в статически деформированном кристалле. Показано, что динамика импульса, распространяющегося под произвольным углом к направлению статической деформации, и эффективных спинов подчиняется модифицированным редуцированным уравнениям Максвелла-Блоха и, если спектр акустического импульса перекрывает квантовые переходы между спиновыми подуровнями, модифицированному уравнению синус-Гордон. Эти уравнения обобщают модели, хорошо известные в теории метода обратной задачи рассеяния и в теории самоиндуцированной прозрачности, и также принадлежат к классу интегрируемых. Анализ солитонных решений показал, что взаимодействие импульсов со средой обнаруживает в данных моделях качественно новые особенности по сравнению со случаями сугубо поперечных или продольных акустических полей.
\end{abstract}

Ключевые слова: солитон, нелинейные интегрируемые уравнения, самоиндуцированная прозрачность.

\section{1. ВВЕДЕНИЕ}

Начиная с 60-х годов прошлого столетия развитие нелинейной оптики в значительной степени стимулирует изучение вопросов интегрируемости уравнений математической физики. Открытие в 1967 г. явления самоиндуцированной прозрачности (СИП) [1] повлекло за собой бурные исследования математической структуры нелинейных уравнений, описывающих этот процесс [2], [3]. Связь уравнений СИП в случае бесконечно узкой спектральной линии и точного резонанса с уравнением синус-Гордон (СГ) сыграла в этих исследованиях существенную роль [2]. Схема Захарова-Шабата в методе обратной задачи рассеяния (MO3P) [4], [5] была

*Российский научный центр "Курчатовский институт", Москва, Россия.

E-mail: barab@newmail.ru

† Томский государственный университет, Томск, Россия. E-mail: n_ustinov@mail.ru 
впервые применена к нелинейному уравнению Шредингера [6], которое описывает распространение оптических импульсов в изотропных нерезонансных средах [7]. Альтернативный подход к изучению СИП, не использующий приближение медленно меняющихся огибающих, а основанный на приближении однонаправленного распространения (ОР) [8], породил редуцированную систему Максвелла-Блоха (РМБ) [9], также интегрируемую с помощью МОЗР.

Следует отметить, что нелинейные оптические явления находили через некоторое время свои аналоги в физической акустике. Так, вскоре после обнаружения оптической СИП был теоретически [10], [11] и экспериментально [10], [12] исследован ее акустический аналог в низкотемпературных кристаллах. В качестве резонансных объектов среды рассматривались парамагнитные примеси с эффективными спинами $S=1 / 2$ [11] и $S=1$ [10], [12], а зеемановское вырождение спиновых подуровней снималось приложением к кристаллу магнитного поля. При этом явление акустической СИП описывалось акустическми уравнениями СИП [10], а в случае точного резонанса - уравнением СГ [11].

Одним из основных направлений развития нелинейной оптики и физической акустики является генерация все более коротких импульсов. Сегодня уже можно говорить о фемтосекундной оптике и пикосекундной акустике как о сформировавшихся областях физической науки [13]. Важно, что столь короткие импульсы вмещают в себя всего несколько (вплоть до одного) периодов колебаний, а примененный к ним термин "предельно короткие импульсы" (ПКИ) можно считать устоявшимся. Описание распространения ПКИ в нелинейных средах потребовало отказа от приближения медленно меняющихся огибающих и использования вместо него приближений ОР, волновой прозначности и спектрального перекрытия (СП) как в оптических [14]-[16], так и в акустических задачах [17].

Акустические импульсы в твердых телах состоят из продольной и поперечной компонент. В парамагнитном кристалле они не только вызывают квантовые переходы между спиновыми подуровнями, но и сдвигают динамическим образом их частоту [18], [19]. Аналогичный эффект имеет место и для оптических импульсов, распространяющихся в среде полярных молекул, которые в стационарных состояниях обладают постоянными дипольными моментами (ПДМ). В простейших случаях динамика ПКИ в такой среде описывается скалярной моделью РМБ с ПДМ, обобщающей уравнения РМБ и также интегрируемой в рамках МОЗР [20]. Более общие векторные оптические и акустические модели были рассмотрены в работах [21]-[24]. В этих условиях описание нелинейной динамики поля и среды тоже проводится с помощью систем уравнений, обладающих свойством интегрируемости.

Как правило, взаимодействие продольно-поперечных акустических импульсов с парамагнитными центрами исследовалось в случае эффективного спина $S=1 / 2$ вследствие относительной простоты модели. В то же время известно, что наиболее сильное взаимодействие с колебаниями кристаллической решетки испытывают парамагнитные ионы с эффективным спином $S=1$ [19], поэтому использование последних наиболее предпочтительно с точки зрения возможных экспериментов. 
В настоящей работе будут выведены и изучены системы уравнений, описывающие нелинейное распространение продольно-поперечных акустических ПКИ в системе эффективных спинов $S=1$. Как и их различные частные случаи, уже изучавшиеся в ряде оптических и акустических задач, они также принадлежат к классу уравнений, интегрируемых с помощью МОЗР.

\section{2. ФИЗИЧЕСКАЯ МОДЕЛЬ}

Рассмотрим кубический кристалл, содержащий парамагнитные примеси с эффективным спином $S=1$ и помещенный в поле однородной статической деформации $\mathcal{E}^{(0)}$. Пусть направление деформации кристалла параллельно оси $z^{\prime}$, совпадающей с одной из его осей симметрии четвертого порядка. Оси $x^{\prime}$ и $y^{\prime}$ направим вдоль двух других осей симметрии кристалла и введем еще одну декартову систему координат, оси $x$ и $z$ которой повернуты относительно $x^{\prime}$ и $z^{\prime}$ на угол $\alpha$, а ось $y$ совпадает с $y^{\prime}$. Будем считать, что акустический импульс распространяется в направлении оси $z$.

Взаимодействие спинов с полями упругой деформации (спин-фононное взаимодействие) осуществляется при $S \geqslant 1$ за счет механизма Ван-Флека [18]: поле деформации создает градиенты внутрикристаллического электрического поля, что, в свою очередь, воздействует на спиновые квантовые подуровни. При этом статическая деформация вызывает вследствие квадрупольного штарк-эффекта расщепление подуровней, снимая вырождение по модулю проекции $S_{z^{\prime}}$ эффективного спина на ось $z^{\prime}$, в результате чего образуются квантовые переходы. Поле деформации акустического импульса способно в данных условиях как индуцировать квадрупольные переходы между спиновыми подуровнями, так и вызывать динамический сдвиг частоты этих переходов.

Для определенности мы выберем статическую деформацию кристалла (сжатие или растяжение) так, что энергия состояния с $S_{z^{\prime}}=0$ меньше энергий состояний с $S_{z^{\prime}}= \pm 1$. Основное состояние парамагнитного иона будем характеризовать порядковым номером 2, а уровни вырожденного возбужденного состояния - номерами 1 и 3. Тогда в представлении собственных функций оператора $\widehat{S}_{z^{\prime}}$ базисные спиновые матрицы будут определены следующим образом [19]:

$$
\widehat{S}_{x^{\prime}}=\frac{1}{\sqrt{2}}\left(\begin{array}{ccc}
0 & 1 & 0 \\
1 & 0 & 1 \\
0 & 1 & 0
\end{array}\right), \quad \widehat{S}_{y}=\frac{i}{\sqrt{2}}\left(\begin{array}{ccc}
0 & 1 & 0 \\
-1 & 0 & 1 \\
0 & -1 & 0
\end{array}\right), \quad \widehat{S}_{z^{\prime}}=\left(\begin{array}{ccc}
-1 & 0 & 0 \\
0 & 0 & 0 \\
0 & 0 & 1
\end{array}\right) .
$$

Пусть импульсы являются пространственно одномерными, т.е. все динамические переменные зависят только от координаты $z$ и времени $t$. В этих условиях гамильтониан спина, находящегося в полях статической и динамической деформаций, имеет вид [18], [19]

$$
\widehat{H}=\hbar \omega_{0} \widehat{S}_{z^{\prime}}^{2}+G_{\|} \mathcal{E}_{z z} \widehat{S}_{z}^{2}+\frac{G_{\perp}\left[\mathcal{E}_{x z}\left(\widehat{S}_{x} \widehat{S}_{z}+\widehat{S}_{z} \widehat{S}_{x}\right)+\mathcal{E}_{y z}\left(\widehat{S}_{y} \widehat{S}_{z}+\widehat{S}_{z} \widehat{S}_{y}\right)\right]}{4},
$$


где $\hbar$ - постоянная Планка, $\omega_{0}=G_{\|} \mathcal{E}^{(0)} / \hbar$ - частота квантового перехода, $G_{\|}=$ $G_{z^{\prime} z^{\prime} z^{\prime} z^{\prime}}=G_{x^{\prime} x^{\prime} x^{\prime} x^{\prime}}=G_{y y y y}$ и $G_{\perp}=G_{x^{\prime} x^{\prime} z^{\prime} z^{\prime}}=G_{x^{\prime} x^{\prime} y y}=G_{y y z^{\prime} z^{\prime}}$ - компоненты тензора взаимодействия спина с продольной (объемной) $\mathcal{E}_{z z}=\partial u_{z} / \partial z$ и поперечными (сдвиговыми) $\mathcal{E}_{x z}=\partial u_{x} / \partial z$ и $\mathcal{E}_{y z}=\partial u_{y} / \partial z$ составляющими поля деформации акустического импульса, $u_{x}, u_{y}$ и $u_{z}$ - соответствующие компоненты вектора локальных смещений u. Матрицы $\widehat{S}_{x}$ и $\widehat{S}_{z}$ связаны с базисными спиновыми матрицами преобразованием поворота [10], [25]:

$$
\widehat{S}_{x}=\widehat{S}_{x^{\prime}} \cos \alpha-\widehat{S}_{z^{\prime}} \sin \alpha, \quad \widehat{S}_{z}=\widehat{S}_{z^{\prime}} \cos \alpha+\widehat{S}_{x^{\prime}} \sin \alpha .
$$

Для гамильтониана $H_{\text {a }}$ свободного акустического поля имеем выражение

$$
H_{\mathrm{a}}=\frac{1}{2} \int\left(\frac{\mathbf{p}^{2}}{\rho}+\rho a_{\|}^{2} \mathcal{E}_{z z}^{2}+\rho a_{\perp}^{2}\left(\mathcal{E}_{x z}^{2}+\mathcal{E}_{y z}^{2}\right)\right) d \mathbf{r},
$$

где $\mathbf{p}$ - вектор плотности импульса, соответствующий динамическим смещениям кристалла, $\rho$ - его равновесная плотность, $a_{\|}$и $a_{\perp}$ - линейные скорости продольного и поперечного звуков. Интегрирование проводится по всему объему кристалла.

Самосогласованную динамику упругого поля и эффективных спинов будем рассматривать, следуя стандартному полуклассическому подходу. В соответствии с ним поведение спинов описывается квантово-механическим уравнением для матрицы плотности $\hat{\rho}$ :

$$
i \hbar \frac{\partial \hat{\rho}}{\partial t}=[\widehat{H}, \hat{\rho}]
$$

а динамика поля деформации акустического импульса - уравнениями Гамильтона для сплошной среды:

$$
\frac{\partial \mathbf{p}}{\partial t}=-\frac{\delta}{\delta \mathbf{u}}\left(H_{\mathrm{a}}+\langle\widehat{\widetilde{H}}\rangle\right), \quad \frac{\partial \mathbf{u}}{\partial t}=\frac{\delta}{\delta \mathbf{p}}\left(H_{\mathrm{a}}+\langle\widehat{\widetilde{H}}\rangle\right),
$$

где $\langle\widehat{\widetilde{H}}\rangle=\int n\langle\widehat{H}\rangle d \mathbf{r}, n-$ концетрация парамагнитных ионов, $\langle\widehat{H}\rangle=\operatorname{Tr}(\hat{\rho} \widehat{H})-$ квантовое среднее $\widehat{H}$.

В уравнении (5) мы пренебрегли необратимой релаксацией, считая, что длительность акустического импульса меньше всех релаксационных времен. Также будем далее предполагать, что матрица плотности имела до прохождения импульса следующий вид:

$$
\left.\hat{\rho}\right|_{t \rightarrow-\infty}=\operatorname{diag}\left(W_{1}, W_{2}, W_{3}\right),
$$

где $W_{1}, W_{2}$ и $W_{3}$ - начальные населенности спиновых подуровней.

Если начальные населенности уровней подчиняются распределению Больцмана (т.е. $\left.W_{3}=W_{1}\right)$ и компонента деформации $\mathcal{E}_{y z}$ отсутствует на входе, то, как было показано в работе [24], она не генерируется внутри кристалла. Поэтому везде ниже мы рассматриваем только одну составляющую сдвиговой деформации $\mathcal{E}_{x z}$. Нетрудно показать, что в этих условиях коэффициенты матрицы плотности будут связаны соотношениями

$$
\rho_{33}=\rho_{11}, \quad \rho_{31}=\rho_{13}, \quad \rho_{32}=-\rho_{12}, \quad \rho_{13}=W_{1}-\rho_{11} .
$$


С учетом данных соотношений уравнение (5) для спиновой динамики можно записать через блоховские переменные

$$
U=-\frac{\rho_{12}+\rho_{21}}{\sqrt{2}}, \quad V=\frac{i\left(\rho_{12}-\rho_{21}\right)}{\sqrt{2}}, \quad W=\frac{1+W_{2}}{4}-\rho_{22}
$$

следующим образом:

$$
\frac{\partial U}{\partial t}=\left(\omega_{0}+\Omega_{2}\right) V, \quad \frac{\partial V}{\partial t}=-\left(\omega_{0}+\Omega_{2}\right) U+\Omega_{1} W, \quad \frac{\partial W}{\partial t}=-\Omega_{1} V,
$$

где мы ввели обозначения

$$
\Omega_{1}=\frac{G_{\perp} \cos (2 \alpha) \mathcal{E}_{x z}+G_{\|} \sin (2 \alpha) \mathcal{E}_{z z}}{\hbar}, \quad \Omega_{2}=\frac{G_{\|} \cos (2 \alpha) \mathcal{E}_{z z}-G_{\perp} \sin (2 \alpha) \mathcal{E}_{x z}}{\hbar} .
$$

Из системы (7) видно, что динамические переменные $\Omega_{1}$ и $\Omega_{2}$, составленные из суперпозиций продольной и поперечной компонент деформации акустического импульса, выполняют при взаимодействии с эффективными спинами строго различные функции. Первая переменная возбуждает квантовые переходы между спиновыми подуровнями, изменяя их населенности, вторая же вызывает динамический сдвиг частоты данных переходов. При $\alpha=0$ и $\alpha= \pm 90^{\circ}$ имеем соответственно $\Omega_{1}= \pm G_{\perp} \mathcal{E}_{x z} / \hbar, \Omega_{2}= \pm G_{\|} \mathcal{E}_{z z} / \hbar$, т.е. при распространении в данных направлениях поперечная компонента импульса вызывает квантовые переходы, а продольная составляющая динамически сдвигает частоту переходов. Если же $\alpha= \pm 45^{\circ}$, то ситуация прямо противоположна. При этом $\Omega_{1}= \pm G_{\|} \mathcal{E}_{z z} / \hbar, \Omega_{2}=\mp G_{\perp} \mathcal{E}_{x z} / \hbar$. В случае же других значений угла $\alpha$ у компонент импульса нет строгого разделения функций. Упомянем в этой связи, что только при углах $\alpha=0, \pm 45^{\circ}, \pm 90^{\circ}$ импульсные составляющие в кубическом кристалле являются строго поперечными или строго продольными; при других углах принято говорить о квазипродольной и квазипоперечных компонентах [26], что обусловлено свойствами симметрии кристалла.

Предположим, что линейные скорости продольной и поперечной акустических волн равны $a_{\|}=a_{\perp}=a$. Тогда из уравнений (6) получим

$$
\begin{aligned}
& \frac{\partial^{2} \Omega_{1}}{\partial t^{2}}-a^{2} \frac{\partial^{2} \Omega_{1}}{\partial z^{2}}=\frac{2 n G^{2}}{\hbar \rho} \frac{\partial^{2}}{\partial z^{2}}(U-q W), \\
& \frac{\partial^{2} \Omega_{2}}{\partial t^{2}}-a^{2} \frac{\partial^{2} \Omega_{2}}{\partial z^{2}}=-\frac{n G^{2}}{\hbar \rho} \frac{\partial^{2}}{\partial z^{2}}\left(q U-2\left(q^{2}+d^{2}\right) W\right),
\end{aligned}
$$

где

$$
G^{2}=G_{\perp}^{2} \cos ^{2}(2 \alpha)+\frac{G_{\|}^{2}}{2} \sin ^{2}(2 \alpha), \quad q=\frac{2 G_{\perp}^{2}-G_{\|}^{2}}{4 G^{2}} \sin (4 \alpha), \quad d=\frac{G_{\perp} G_{\|}}{\sqrt{2} G^{2}} .
$$

Отметим, что линейные скорости акустических волн равны, если компоненты тензора упругих постоянных в упруго-изотропном кристалле удовлетворяют соотношениям Коши [27]. 
При выполнении неравенства

$$
\eta=\frac{n\left(G_{\perp}^{2}+G_{\|}^{2}\right)}{\hbar \omega_{0} \rho a^{2}} \ll 1,
$$

что обычно имеет место в парамагнитных кристаллах [26], правые части уравнений (9), (10) много меньше каждого из слагаемых в левых частях этих же уравнений. В этом случае можно пренебречь волной, отраженной от парамагнитных примесей, и применить к рассматриваемым уравнениям приближение ОР [8]. Введем для этого "локальное" время $\tau=t-z / a$ и "медленную" координату $\zeta=\eta z$. Тогда

$$
\frac{\partial}{\partial t}=\frac{\partial}{\partial \tau}, \quad \frac{\partial}{\partial z}=-\frac{1}{a} \frac{\partial}{\partial \tau}+\eta \frac{\partial}{\partial \zeta} .
$$

Отбрасывая слагаемые, пропорциональные $\eta^{2}$, в левых частях уравнений (9), (10) будем иметь

$$
\frac{\partial^{2}}{\partial z^{2}} \approx \frac{1}{a^{2}} \frac{\partial^{2}}{\partial \tau^{2}}-2 \frac{\eta}{a} \frac{\partial^{2}}{\partial \tau \partial \zeta}
$$

а в правых -

$$
\frac{\partial^{2}}{\partial z^{2}} \approx \frac{1}{a^{2}} \frac{\partial^{2}}{\partial \tau^{2}}
$$

Учитывая также уравнения (7) и нулевые значения поля импульса и его производных на бесконечности, получим после интегрирования по $\tau$ и возвращения к исходным переменным $z$ и $t$

$$
\begin{aligned}
& \frac{\partial \Omega_{1}}{\partial z}+\frac{1}{a} \frac{\partial \Omega_{1}}{\partial t}=\beta\left(\omega_{0}+\Omega_{2}+q \Omega_{1}\right) V \\
& \frac{\partial \Omega_{2}}{\partial z}+\frac{1}{a} \frac{\partial \Omega_{2}}{\partial t}=-\beta\left[q\left(\omega_{0}+\Omega_{2}+q \Omega_{1}\right)+d^{2} \Omega_{1}\right] V,
\end{aligned}
$$

где $\beta=n G^{2} /\left(\hbar \rho a^{3}\right)$.

Отметим, что из формул (12), (13) следует интеграл движения

$$
\left(\omega_{0}+\Omega_{2}+q \Omega_{1}\right)^{2}+d^{2} \Omega_{1}^{2}=\omega_{0}^{2}(1+f(\tau))^{2},
$$

где функция $f(\tau)$ определяется условиями на входе импульса в среду. Важным следствием существования данного интеграла являются ограничения на области значений переменных $\Omega_{1}$ и $\Omega_{2}$. Так, если $f(\tau)=0$, то получаем

$$
\left|\Omega_{1}\right| \leqslant \frac{1}{|d|}, \quad-\omega_{0}\left(1+\sqrt{1+\frac{q^{2}}{d^{2}}}\right) \leqslant \Omega_{2} \leqslant \omega_{0}\left(-1+\sqrt{1+\frac{q^{2}}{d^{2}}}\right) .
$$

С помощью замены переменных $\left\{z, t, \Omega_{1}, \Omega_{2}\right\} \rightarrow\left\{z^{\prime}, t^{\prime}, \Omega_{1}^{\prime}, \Omega_{2}^{\prime}\right\}$, где

$$
z^{\prime}=z, \quad t^{\prime}=\int_{0}^{\tau}\left|1+f\left(\tau^{\prime}\right)\right| d \tau^{\prime}+\frac{z}{a}, \quad \Omega_{1}^{\prime}=\frac{\Omega_{1}}{|1+f(\tau)|}, \quad \Omega_{2}^{\prime}=\frac{\omega_{0}+\Omega_{2}}{|1+f(\tau)|}-\omega_{0},
$$

рассмотрение можно свести без потери общности к случаю $f(\tau)=0$. 
Уравнения (7), (12), (13) описывают динамику продольно-поперечных акустических импульсов в деформированном кристалле, содержащем парамагнитные примеси с эффективным спином $S=1$. Ниже данную систему будем называть модифицированными уравнениями РМБ (МРМБ). Смысл этого названия станет ясен в разделе 4.

Уравнения МРМБ представляют интерес как с точки зрения их физических приложений, так и благодаря математическим свойствам. В случае $d=q=0$ и $\Omega_{2}=0$ они, очевидно, совпадают с уравнениями РМБ для изотропной двухуровневой среды [9]. Если $d=0, q \neq 0$, то можно положить $\Omega_{2}=-q \Omega_{1}$. Возникающая при этом система сводится к уравнениям РМБ с ПДМ, интегрируемость которых с помощью МОЗР была установлена в работе [20]. Эти уравнения исследовались численно и аналитически в работах [28] и [29], соответственно, где было показано, что характер взаимодействия импульса со средой существенным образом зависит от знака его полярности. Также данные уравнения в этом случае определяют динамику квазипродольного звука в деформированном парамагнитном кристалле с эффективным спином $S=1$ [30]. Кроме того, при $q=0, d \neq 0$ уравнения МРМБ переходят в двухкомпонентные уравнения РМБ, которые описывают распространение векторных электромагнитных импульсов в оптически одноосной среде. Солитонные решения этих уравнений также обнаружили асимметрию по полярности одной из компонент сигнала [31]. Наконец, уравнения (7), (12) и (13) эквивалентны системе волновых и материальных уравнений, которая была выведена в работе [22] при рассмотрении динамики продольно-поперечных акустических импульсов в парамагнитном кристалле с эффективным спином $S=1 / 2$ в присутствии внешнего магнитного поля. Также в этой работе было установлено, что данная система принадлежит к классу интегрируемых и имеет пару Лакса, коэффициенты которой являются многозначными функциями спектрального параметра. Ниже мы покажем, что у уравнений МРМБ есть пара Лакса с однозначной зависимостью коэффициентов от спектрального параметра, и построим для нее солитонные решения.

Заметим, что использование приближения медленно меняющихся огибающих в данной физической задаче тоже приводит к интегрируемой системе уравнений [24], которая была подробно изучена в связи с ее приложениями в когерентной оптике в работе [23].

\section{3. МОДИФИЦИРОВАННОЕ УРАВНЕНИЕ СГ}

В этом разделе мы получим еще одно интегрируемое с помощью МОЗР уравнение, применив приближение СП [14] к уравнениям МРМБ. Пусть длительность импульса $\tau_{\mathrm{p}}$ и частота $\omega_{0}$ квантового перехода таковы, что выполняется неравенство

$$
\left(\omega_{0} \tau_{\mathrm{p}}\right)^{2} \ll 1
$$

Физический смысл данного неравенства состоит в том, что спектральная ширина $\Delta \omega \sim 1 / \tau_{\mathrm{p}}$ акустического импульса превышает частоту квантового перехода между 
спиновыми подуровнями. В работах [14], [16] приближение СП использовалось при рассмотрении подобных задач для нелинейной динамики оптических импульсов.

Считая, что величины $\omega_{0}$ и $\Omega_{2}$ одного порядка, и учитывая неравенство (16), пренебрежем в уравнениях (7) слагаемыми, содержащими множитель $\omega_{0}+\Omega_{2}$. Тогда, приняв во внимание начальное условие для $\hat{\rho}$, получим из уравнений (7)

$$
V=W_{0} \sin \theta, \quad W=W_{0} \cos \theta,
$$

где $\theta=\int_{-\infty}^{t} \Omega_{1} d t^{\prime}, W_{0}=\left(1-3 W_{2}\right) / 4$ (при термодинамическом равновесии $\left.W_{0}<0\right)$.

Подставив первое из соотношений (17) в правую часть уравнения (12), придем после использования выражения (14) к модифицированному уравнению СГ (МСГ)

$$
\frac{\partial^{2} \theta}{\partial z \partial T}=-b \sqrt{1-\tau_{\mathrm{c}}^{2}\left(\frac{\partial \theta}{\partial T}\right)^{2}} \sin \theta
$$

где $T=\int_{0}^{\tau}\left|1+f\left(\tau^{\prime}\right)\right| d \tau^{\prime}, b=-\beta \omega_{0} W_{0}, \tau_{\mathrm{c}}=|d| / \omega_{0}$.

Уравнение (18) принадлежит к классу интегрируемых с помощью МОЗР. Впервые оно появилось при изучении свойств преобразований Беклунда уравнения СГ в работах [32], и дальнейшие его исследования велись в этом же направлении [33]. Недавно было показано, что МСГ в приближении СП описывает динамику предельно короткого электромагнитного импульса в системе несимметричных квантовых объектов типа квантовых ям или нитей [34], [35]. Заметим, что в нашем случае для описания распространения акустического импульса с помощью МСГ он должен обладать продольно-поперечной структурой. Если хотя бы одна из компонент поля деформации не взаимодействует с парамагнитными примесями $\left(G_{\|}=0\right.$ или $\left.G_{\perp}=0\right)$, то $\tau_{\mathrm{c}}=0$, и уравнение (18) переходит в уравнение СГ.

В разделе 5 мы будем исследовать особенности динамики полей деформации и спинов, основываясь на солитонных решениях уравнений МРМБ и МСГ, а в заключение данного раздела сделаем замечание, касающееся области применимости уравнения МСГ. Использованное в разделе 2 приближение ОР предполагает малость правых частей уравнений (9) и (10) по сравнению с каждым из слагаемых в их левых частях. Тогда из уравнения (9) имеем

$$
\left|\Omega_{1}\right| \gg \frac{n G^{2}}{\hbar \rho a^{2}}|U| .
$$

Из уравнений (7) и (17) следует, что $U \sim \omega_{0} \tau_{\mathrm{p}} V \sim \omega_{0} \tau_{\mathrm{p}} W_{0}$. Полагая $W_{0} \sim 1$, $\left|\Omega_{1}\right| \sim|\theta| / \tau_{\mathrm{p}} \sim 1 / \tau_{\mathrm{p}}$ и принимая во внимание выражение для $\eta$ из неравенства (11), перепишем последнее неравенство в виде

$$
\eta\left(\omega_{0} \tau_{\mathrm{p}}\right)^{2} \ll 1 .
$$

Это условие тоже позволяет применить приближение ОР к уравнениям (9) и (10) и, как видно из неравенства (16), является значительно менее жестким, нежели (11). Таким образом, описание динамики акустических импульсов с помощью уравнения (18) в данной задаче не предполагает, в отличие от системы МРМБ, обязательного выполнения неравенства (11). 


\section{4. ПАРЫ ЛАКСА И ПРЕОБРАЗОВАНИЯ ДАРБУ}

Система МРМБ (7), (12), (13) допускает представление в виде условия нулевой кривизны

$$
\frac{\partial \hat{L}}{\partial z}-\frac{\partial \hat{A}}{\partial t}+[\hat{L}, \hat{A}]=0
$$

в котором матрицы $\hat{L}$ и $\hat{A}$ имеют вид

$$
\hat{L}=\frac{1}{2}\left(\begin{array}{cc}
L_{11} & i \lambda L_{12} \\
i \lambda L_{12}^{*} & -L_{11}
\end{array}\right), \quad \hat{A}=\frac{\beta \tau_{r}}{2\left(1+\tau_{r}^{-2}-\lambda^{2}\right)}\left(\begin{array}{cc}
A_{11} & i \lambda A_{12} \\
i \lambda A_{12}^{*} & -A_{11}
\end{array}\right)-\frac{\hat{L}}{a} .
$$

Здесь $\lambda$ - спектральный параметр,

$$
\begin{gathered}
L_{11}=\frac{\omega_{0}+\Omega_{2}+q \Omega_{1}}{\tau_{\mathrm{r}}}+q \tau_{\mathrm{r}} \Omega_{1}, \quad L_{12}=\omega_{0}+\Omega_{2}+(q+i d) \Omega_{1}, \\
A_{11}=\frac{W}{\tau_{\mathrm{r}}^{2}}-i d \tau_{\mathrm{r}} \lambda^{2} V+q\left(1+\tau_{\mathrm{r}}^{-2}\right) U, \quad A_{12}=\frac{W}{\tau_{\mathrm{r}}}+\frac{(q+i d) U}{\tau_{\mathrm{r}}}+\left(q-\frac{i d}{\tau_{\mathrm{r}}^{2}}\right) V,
\end{gathered}
$$

$\tau_{\mathrm{r}}-$ корень уравнения

$$
q^{2} \tau_{\mathrm{r}}^{4}+\left(1+q^{2}-d^{2}\right) \tau_{\mathrm{r}}^{2}-d^{2}=0
$$

Уравнение (19) является условием совместности переопределенной системы линейных уравнений (пары Лакса)

$$
\begin{aligned}
& \frac{\partial \psi}{\partial t}=\hat{L} \psi, \\
& \frac{\partial \psi}{\partial z}=\hat{A} \psi
\end{aligned}
$$

где $\psi=\psi(t, z, \lambda)=\left(\psi_{1}, \psi_{2}\right)^{\mathrm{T}}$ - векторное решение пары Лакса. В отличие от пары Лакса, найденной в работе [22], коэффициенты системы (21) являются однозначными функциями спектрального параметра.

Уравнение МСГ (18) также можно записать в виде условия нулевой кривизны

$$
\frac{\partial \hat{L}_{\mathrm{M}}}{\partial z}-\frac{\partial \hat{A}_{\mathrm{M}}}{\partial T}+\left[\hat{L}_{\mathrm{M}}, \hat{A}_{\mathrm{M}}\right]=0
$$

где

$$
\begin{gathered}
\hat{L}_{\mathrm{M}}=\frac{1}{2}\left(\begin{array}{cc}
i u & \varkappa Q^{*} \\
\varkappa Q & -i u
\end{array}\right), \quad \hat{A}_{\mathrm{M}}=-\frac{b}{2 \varkappa}\left(\begin{array}{cc}
-i \varkappa \tau_{\mathrm{c}} \sin \theta & e^{i \theta} \\
e^{-i \theta} & i \varkappa \tau_{\mathrm{c}} \sin \theta
\end{array}\right), \\
Q=\sqrt{1-\tau_{\mathrm{c}}^{2} u^{2}}+i \tau_{\mathrm{c}} u, \quad u=\frac{\partial \theta}{\partial T},
\end{gathered}
$$

$\varkappa$ - спектральный параметр. Соответствующая пара Лакса имеет вид

$$
\begin{aligned}
\frac{\partial \xi}{\partial T} & =\hat{L}_{\mathrm{M}} \xi \\
\frac{\partial \xi}{\partial z} & =\hat{A}_{\mathrm{M}} \xi
\end{aligned}
$$

где $\xi=\xi(T, z, \varkappa)=\left(\xi_{1}, \xi_{2}\right)^{\mathrm{T}}-$ векторное решение. 
Переопределенные системы (21) и (23) связаны с парами Лакса соответственно системы РМБ с ПДМ [20] и уравнения СГ калибровочными преобразованиями [5]. Таким образом, системы $(7),(12),(13)$ с $d \neq 0$ и с $d=0$ калибровочно-эквивалентны друг другу. То же самое справедливо и для уравнений МСГ и СГ. По аналогии мы тоже называем уравнения (7), (12), (13) модифицированными. Заметим в этой связи, что пары Лакса (21) системы МРМБ, которые соответствуют различным корням уравнения (20), связаны, очевидно, калибровочными преобразованиями.

Отмеченная выше калибровочная эквивалентность дает возможность модифицировать МОЗР, развитый для уравнений РМБ и СГ, для рассматриваемых здесь случаев. Так, аппарат МОЗР был применен к системе МРМБ при $q=0$ в работе [22]. Однако известно, что солитонные решения интегрируемых уравнений можно найти не только с помощью МОЗР, но и используя более простые алгебраические методы. В настоящей работе к построению решений будет привлечена техника преобразования Дарбу (ПД) [36].

Пусть $\varphi=\left(\varphi_{1}, \varphi_{2}\right)^{\mathrm{T}}$ - векторное решение переопределенной системы $(21)$ при $\lambda=\mu$. Пара Лакса (21) ковариантна относительно ПД $\left\{\psi_{1}, \psi_{2}, \Omega_{1}, \Omega_{2}, U, V, W\right\} \rightarrow$ $\left\{\tilde{\psi}_{1}, \tilde{\psi}_{2}, \widetilde{\Omega}_{1}, \widetilde{\Omega}_{2}, \widetilde{U}, \widetilde{V}, \widetilde{W}\right\}$, где преобразованные величины определены следующим образом:

$$
\begin{gathered}
\tilde{\psi}_{1}=\sqrt{\frac{\delta_{2}}{\delta_{1}}}\left(\mu \psi_{1}-\frac{\lambda \varphi_{1} \psi_{2}}{\varphi_{2}}\right), \quad \tilde{\psi}_{2}=\sqrt{\frac{\delta_{1}}{\delta_{2}}}\left(\mu \psi_{2}-\frac{\lambda \varphi_{2} \psi_{1}}{\varphi_{1}}\right) \\
\widetilde{\Omega}_{1}=\Omega_{1}-\frac{\partial}{\partial t}\left[\frac{\ln \delta_{1}}{s_{+}}-\frac{\ln \delta_{2}}{s_{-}}\right], \quad \widetilde{\Omega}_{2}=\Omega_{2}+\frac{\partial}{\partial t}\left[\frac{q-i d}{s_{+}} \ln \delta_{1}-\frac{q+i d}{s_{-}} \ln \delta_{2}\right], \\
\widetilde{U}=U-\frac{i}{8 d}\left(\frac{\partial}{\partial z}+\frac{1}{a} \frac{\partial}{\partial t}\right)\left[\frac{q-i d}{s_{+}} \ln \delta_{1}+\frac{q+i d}{s_{-}} \ln \delta_{2}\right] \\
\widetilde{V}=-V+\frac{i}{8 d}\left(\frac{\partial}{\partial z}+\frac{1}{a} \frac{\partial}{\partial t}\right) \ln \frac{\delta_{1} \varphi_{2}^{2}}{\delta_{2} \varphi_{1}^{2}}, \quad \widetilde{W}=W-\frac{i}{8 d}\left(\frac{\partial}{\partial z}+\frac{1}{a} \frac{\partial}{\partial t}\right)\left[\frac{\ln \delta_{1}}{s_{+}}+\frac{\ln \delta_{2}}{s_{-}}\right] .
\end{gathered}
$$

Здесь мы использовали обозначения

$$
\delta_{1}=\frac{s_{+}}{d} \frac{\varphi_{1}}{\varphi_{2}}-\mu, \quad \delta_{2}=\frac{s_{-}}{d} \frac{\varphi_{2}}{\varphi_{1}}-\mu, \quad s_{ \pm}=\frac{q \tau_{\mathrm{r}}^{2} \pm i d}{\tau_{\mathrm{r}}} .
$$

Равенства (25)-(27) определяют новое (“одетое") решение уравнений (7), (12), (13). В пределе $q \rightarrow 0, d \rightarrow 0$ и, следовательно, $\tau_{\mathrm{r}} \rightarrow 0$ формулы $(24)-(27)$ переходят в ПД для системы РМБ [36]. Вследствие указанной в этом разделе калибровочной эквивалентности преобразование $(24)-(27)$ в случае $d \neq 0$ есть результат последовательного проведения ПД при $d=0$ и калибровочного преобразования.

Пара Лакса (23) ковариантна относительно ПД $\left\{\xi_{1}, \xi_{2}, \theta\right\} \rightarrow\left\{\tilde{\xi}_{1}, \tilde{\xi}_{2}, \tilde{\theta}\right\}$ вида [35]

$$
\begin{gathered}
\tilde{\xi}_{1}=e^{i(\tilde{\theta}-\theta) / 2}\left(\nu \xi_{1}-\frac{\varkappa \chi_{1} \xi_{2}}{\chi_{2}}\right), \quad \tilde{\xi}_{2}=e^{i(\theta-\tilde{\theta}) / 2}\left(\nu \xi_{2}-\frac{\varkappa \chi_{2} \xi_{1}}{\chi_{1}}\right), \\
\tilde{\theta}=\theta+i \ln \frac{\chi_{1}^{2}-\nu \tau_{\mathrm{c}} \chi_{1} \chi_{2}}{\chi_{2}^{2}-\nu \tau_{\mathrm{c}} \chi_{1} \chi_{2}}
\end{gathered}
$$


где $\chi_{1}$ и $\chi_{2}$ - компоненты решения $\chi$ системы $(23)$ при $\varkappa=\nu$.

Соотношение (29) дает новое ("одетое") решение уравнения (18). Формулы ПД для уравнения СГ [36] следуют из соотношений (28) и (29) при $\tau_{\mathrm{c}}=0$. Подобно предыдущему случаю, преобразование (28), (29) является результатом последовательного проведения ПД уравнения СГ и калибровочного преобразования.

Равенства (24) и (28) определяют компоненты векторных решений пар Лакса, которые соответствуют "одетым" решениям уравнений МРМБ и МСГ. Это обстоятельство позволяет выполнить итерации ПД и получить бесконечную иерархию решений данных уравнений. Так, $N$-кратное проведение преобразования (28), (29) дает следующее выражение для "одетого" решения уравнения МСГ:

$$
\tilde{\theta}=\theta+i \ln \frac{\Delta^{(1)}}{\Delta^{(2)}}
$$

где

$$
\Delta^{(1)}=\left|\begin{array}{ccccc}
\chi_{1}^{(1)} & \nu_{1} \chi_{2}^{(1)} & \nu_{1}^{2} \chi_{1}^{(1)} & \ldots & \nu_{1}^{N-1} \chi_{K}^{(1)} \\
\chi_{1}^{(2)} & \nu_{2} \chi_{2}^{(2)} & \nu_{2}^{2} \chi_{1}^{(2)} & \ldots & \nu_{2}^{N-1} \chi_{K+}^{(2)} \\
\chi_{1}^{(3)} & \nu_{3} \chi_{2}^{(3)} & \nu_{3}^{2} \chi_{1}^{(3)} & \ldots & \nu_{3}^{N-1} \chi_{K_{+}}^{(3)} \\
\vdots & \vdots & \vdots & \ddots & \vdots \\
\chi_{1}^{(N)} & \nu_{N} \chi_{2}^{(N)} & \nu_{N}^{2} \chi_{1}^{(N)} & \ldots & \nu_{N}^{N-1} \chi_{K_{+}(N)}^{(N)}
\end{array}\right|\left|\begin{array}{ccccc}
\tau_{\mathrm{c}}^{N} & \tau_{\mathrm{c}}^{N-1} & \tau_{\mathrm{c}}^{N-2} & \ldots & 1 \\
\chi_{1}^{(1)} & \nu_{1} \chi_{2}^{(1)} & \nu_{1}^{2} \chi_{1}^{(1)} & \ldots & \nu_{1}^{N} \chi_{K}^{(1)} \\
\chi_{1}^{(2)} & \nu_{2} \chi_{2}^{(2)} & \nu_{2}^{2} \chi_{1}^{(2)} & \ldots & \nu_{2}^{N} \chi_{K}^{(2)} \\
\vdots & \vdots & \vdots & \ddots & \vdots \\
\chi_{1}^{(N)} & \nu_{N} \chi_{2}^{(N)} & \nu_{N}^{2} \chi_{1}^{(N)} & \ldots & \nu_{N}^{N} \chi_{K}^{(N)}
\end{array}\right|
$$

$\chi_{1}^{(L)}$ и $\chi_{2}^{(L)}-$ компоненты векторного решения $\chi^{(L)}$ пары Лакса $(23)$ при $\varkappa=\nu_{L}$, $L=1, \ldots, N ; \Delta^{(1)}$ переходит в $\Delta^{(2)}$ при замене нижних индексов $1 \leftrightarrow 2$ у компонент векторных решений; $K_{ \pm}=\left(3 \pm(-1)^{N}\right) / 2$.

\section{5. СОЛИТОННЫЕ РЕШЕНИЯ}

Используем формулы ПД, представленные в предыдущем разделе, для нахождения солитонных решений рассматриваемых уравнений. Возьмем в качестве исходного решения уравнений МРМБ нулевой фон: $\Omega_{1}=\Omega_{2}=0, U=V=0, W=W_{0}$. Выражения для компонент решения $\varphi$ пары Лакса (21) с $\lambda=\mu$ имеют вид

$$
\varphi_{1}=c_{1} e^{\gamma / 2}+c_{2} e^{-\gamma / 2}, \quad \varphi_{2}=i c_{1} \frac{\tau_{\mathrm{s}}-\tau_{\mathrm{r}}}{\mu \tau_{\mathrm{s}} \tau_{\mathrm{r}}} e^{\gamma / 2}+i c_{2} \frac{\tau_{\mathrm{s}}+\tau_{\mathrm{r}}}{\mu \tau_{\mathrm{s}} \tau_{\mathrm{r}}} e^{-\gamma / 2},
$$

где $c_{1}$ и $c_{2}$ - произвольные постоянные,

$$
\gamma=\frac{\omega_{0}(t-z / v)}{\tau_{\mathrm{s}}}, \quad \tau_{\mathrm{s}}^{-1}=\sqrt{\tau_{\mathrm{r}}^{-2}-\mu^{2}}, \quad v=a\left(1-\frac{a \beta}{1+\tau_{\mathrm{s}}^{-2}} \frac{W_{0}}{\omega_{0}}\right)^{-1} .
$$

В дальнейшем мы будем полагать, используя сдвиги по независимым переменным, постоянные $c_{1}$ и $c_{2}$ равными заданным величинам, выбранным из соображений удобства и обеспечивающим вещественность и несингулярность солитонного решения, которое будет построено с помощью ПД. Также для определенности будем считать, что $\tau_{\mathrm{r}}$ - положительный корень уравнения (20). 
Подставив выражения (31) в соотношения (25)-(27), получим односолитонное решение уравнений МРМБ. Если свободный параметр $\tau_{\mathrm{s}}\left(\tau_{\mathrm{s}} \in \mathbb{R}\right)$ односолитонного решения удовлетворяет условию $\tau_{\mathrm{s}}>\tau_{\mathrm{r}}$ (т.е. $\mu \in \mathbb{R},|\mu|<1 / \tau_{\mathrm{r}}$ ), его можно записать следующим образом:

$$
\begin{gathered}
\Omega_{1}=-2 \omega_{0} \frac{r \sqrt{1-\tau_{\mathrm{r}}^{2} / \tau_{\mathrm{s}}^{2}} \operatorname{ch} \gamma+q}{\tau_{\mathrm{s}}^{2} \Delta_{1}}, \quad \Omega_{2}=2 \omega_{0} \frac{r q \sqrt{1-\tau_{\mathrm{r}}^{2} / \tau_{\mathrm{s}}^{2}} \operatorname{ch} \gamma+q^{2}-d^{2} / \tau_{\mathrm{s}}^{2}}{\tau_{\mathrm{s}}^{2} \Delta_{1}}, \\
U=-4 W_{0} \frac{r \sqrt{1-\tau_{\mathrm{r}}^{2} / \tau_{\mathrm{s}}^{2}} \operatorname{ch} \gamma+q\left(1+\tau_{\mathrm{s}}^{-2}\right)}{\left(1+\tau_{\mathrm{s}}^{2}\right) \Delta_{1}}, \quad V=4 W_{0} \frac{r \sqrt{1-\tau_{\mathrm{r}}^{2} / \tau_{\mathrm{s}}^{2}} \operatorname{sh} \gamma}{\tau_{\mathrm{s}}\left(1+\tau_{\mathrm{s}}^{2}\right) \Delta_{1}} \\
W=W_{0}-\frac{2 W_{0}}{\tau_{\mathrm{s}}^{2}\left(1+\tau_{\mathrm{s}}^{2}\right) \Delta_{1}},
\end{gathered}
$$

где

$$
\begin{gathered}
\Delta_{1}=\left[r\left(\operatorname{ch} \gamma-\frac{\tau_{\mathrm{r}}}{\tau_{\mathrm{s}}} \operatorname{sh} \gamma\right)+q \sqrt{\left.1-\frac{\tau_{\mathrm{r}}^{2}}{\tau_{\mathrm{s}}^{2}}\right]\left[r\left(\operatorname{ch} \gamma+\frac{\tau_{\mathrm{r}}}{\tau_{\mathrm{s}}} \operatorname{sh} \gamma\right)+q \sqrt{1-\frac{\tau_{\mathrm{r}}^{2}}{\tau_{\mathrm{s}}^{2}}}\right]}\right. \\
r= \pm \sqrt{\frac{d^{2}}{\tau_{\mathrm{r}}^{2} \tau_{\mathrm{s}}^{2}}+q^{2}} .
\end{gathered}
$$

При $0<\tau_{\mathrm{s}}<\tau_{\mathrm{r}}(i \mu \in \mathbb{R})$ односолитонное решение имеет вид

$$
\begin{gathered}
\Omega_{1}=2 \omega_{0} \frac{r \sqrt{\tau_{\mathrm{r}}^{2} / \tau_{\mathrm{s}}^{2}-1} \operatorname{sh} \gamma-q}{\tau_{\mathrm{s}}^{2} \Delta_{2}}, \quad \Omega_{2}=-2 \omega_{0} \frac{r q \sqrt{\tau_{\mathrm{r}}^{2} / \tau_{\mathrm{s}}^{2}-1} \operatorname{sh} \gamma-q^{2}+d^{2} / \tau_{\mathrm{s}}^{2}}{\tau_{\mathrm{s}}^{2} \Delta_{2}} \\
U=4 W_{0} \frac{r \sqrt{\tau_{\mathrm{r}}^{2} / \tau_{\mathrm{s}}^{2}-1} \operatorname{sh} \gamma-q\left(1+\tau_{\mathrm{s}}^{-2}\right)}{\left(1+\tau_{\mathrm{s}}^{2}\right) \Delta_{2}}, \quad V=-4 W_{0} \frac{r \sqrt{\tau_{\mathrm{r}}^{2} / \tau_{\mathrm{s}}^{2}-1} \operatorname{ch} \gamma}{\tau_{\mathrm{s}}\left(1+\tau_{\mathrm{s}}^{2}\right) \Delta_{2}} \\
W=W_{0}-\frac{2 W_{0}}{\tau_{\mathrm{s}}^{2}\left(1+\tau_{\mathrm{s}}^{2}\right) \Delta_{2}}
\end{gathered}
$$

где

$$
\Delta_{2}=\left[r\left(\frac{\tau_{\mathrm{r}}}{\tau_{\mathrm{s}}} \operatorname{ch} \gamma-\operatorname{sh} \gamma\right)-q \sqrt{\frac{\tau_{\mathrm{r}}^{2}}{\tau_{\mathrm{s}}^{2}}-1}\right]\left[r\left(\frac{\tau_{\mathrm{r}}}{\tau_{\mathrm{s}}} \operatorname{ch} \gamma+\operatorname{sh} \gamma\right)+q \sqrt{\frac{\tau_{\mathrm{r}}^{2}}{\tau_{\mathrm{s}}^{2}}-1}\right] .
$$

Данное решение описывает распространение продольно-поперечного акустического импульса. Поскольку его скорость $v$ не зависит от корня $\tau_{\text {r }}$ уравнения $(20)$, то формулы ПД (25)-(27) с решениями пар Лакса, соответствующими другим корням данного уравнения, не дадут новых односолитонных решений системы МРМБ. Отметим, что солитонное решение, найденное в работе [20], соответствует случаю $q=0$.

При $\tau_{\mathrm{s}}>\tau_{\mathrm{r}}$ форма импульса существенно зависит от знаков $r$ и $q$. Если знаки одинаковы, то компонента $\Omega_{1}$ имеет один горб, знак которого такой же, как у $q$, a $\Omega_{2}$ мало отличается от нуля (см. кривые, изображенные на рис. 1а, б сплошными линиями). Возбуждение среды в таких условиях мало (рис. 1в). С увеличением $\tau_{\mathrm{s}}$ амплитуда поля деформации и степень возбуждения среды убывают. Характер зависимости скорости солитона, его амплитуды и поведения среды от длительности солитона при $\tau_{\mathrm{c}} \gg \tau_{\mathrm{r}}$ является таким же, как и в случае солитонов уравнения РМБ. 


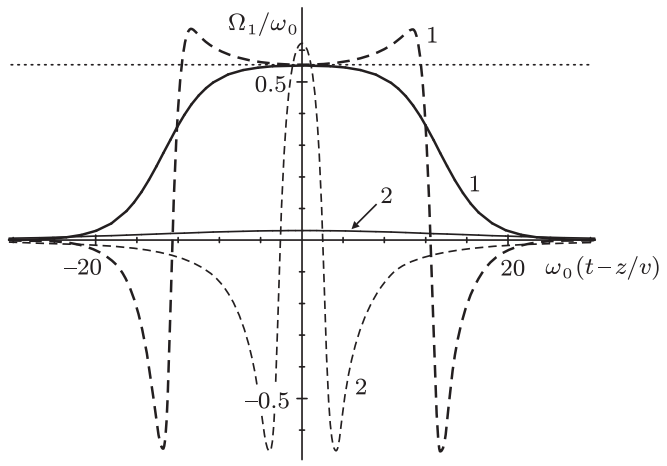

a

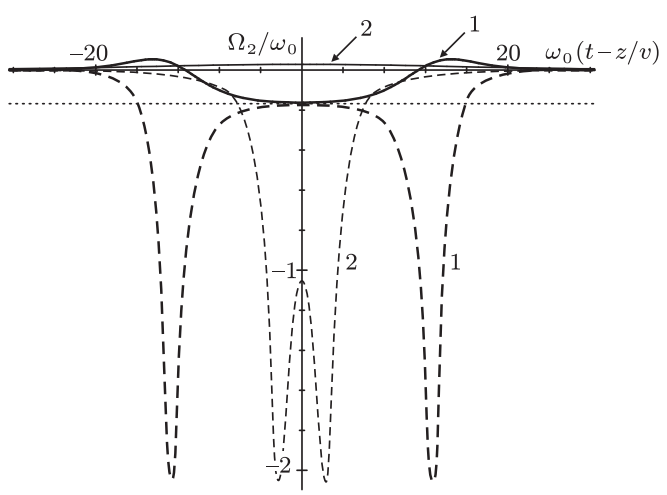

б

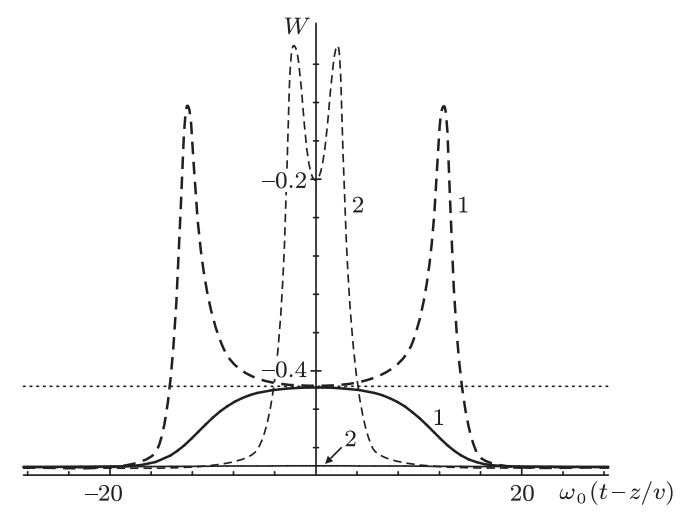

B

Рис. 1. Профили компонент поля деформации (а), (б) и инверсии населенности (в) в случае $\tau_{\mathrm{s}}>\tau_{\mathrm{r}}$ при $q=0.5, d=1.5, W_{0}=-0.5$ для $r q>0$, $\tau_{\mathrm{s}}=2.3677$ и $\tau_{\mathrm{s}}=10$ (сплошные линии 1 и 2 , соответственно); для $r q<0$, $\tau_{\mathrm{s}}=2.3677$ и $\tau_{\mathrm{s}}=10$ (штриховые линии 1 и 2 , соответственно). Пунктирные прямые соответствуют значениям величин в центре импульса в пределе $\tau_{\mathrm{s}} \rightarrow \tau_{\mathrm{r}} \approx 2.3676$. 
При разных знаках у $r$ и $q$ поле $\Omega_{1}$ имеет четыре горба разной полярности (или три, если $\tau_{\mathrm{s}} \gg \tau_{\mathrm{r}}$ ), а $\Omega_{2}$ состоит из двух горбов отрицательной полярности (штриховые линии на рис. 1а, б). Так как частота квантового перехода динамически понижается при прохождении импульса за счет компоненты $\Omega_{2}$ (см. первое и второе из уравнений (7)), то среда возбуждается в этом случае гораздо сильнее.

В пределе $\tau_{\mathrm{s}} \rightarrow \infty$ решение (32)-(34) рационально зависит от $t$ и $z$. В частности, для переменных $\Omega_{1}, \Omega_{2}$ и $W$ получим следующие выражения:

$$
\Omega_{1}=4 \omega_{0} q \frac{Q-d^{2}}{R_{+} R_{-}}, \quad \Omega_{2}=-4 \omega_{0} q^{2} \frac{Q+d^{2}}{R_{+} R_{-}}, \quad W=W_{0}-\frac{8 W_{0}}{R_{+} R_{-}},
$$

где

$Q=q^{2} \omega_{0}^{2}\left(t-\frac{z}{v_{\mathrm{r}}}\right)^{2}+q^{2}+1, \quad R_{ \pm}=q^{2}\left(\omega_{0}\left(t-\frac{z}{v_{\mathrm{r}}}\right) \pm \tau_{\mathrm{r}}\right)^{2}+\frac{d^{2}}{\tau_{\mathrm{r}}^{2}}, \quad v_{r}=\frac{a}{\left(1-\frac{a \beta W_{0}}{\omega_{0}}\right)}$.

Амплитуды поля деформации и изменения инверсии населенности, а также длительность солитона остаются при этом конечными (ср. штриховые линии 2 и сплошные кривые 2 на рис. 1). Отметим, что существование рациональных и рационально убывающих электромагнитных импульсов является отличительной чертой анизотропных сред [29]-[31], [37].

Если $\tau_{\mathrm{s}}<\tau_{\mathrm{r}}$, то случаи одинаковых и противоположных знаков $r$ и $q$ переходят друг в друга при замене $\gamma \rightarrow-\gamma$. Компонента $\Omega_{1}$ имеет здесь два несимметричных горба разной полярности, а $\Omega_{2}$ - один горб отрицательной полярности (см. рис. $2 \mathrm{a}$, б). Знак площади компоненты $\Omega_{1}$ совпадает со знаком $q$, как и при $\tau_{\mathrm{s}}>\tau_{\mathrm{r}}$. Возбуждение среды тоже оказывается достаточно сильным (рис. 2в). Отметим, что в пределе $\tau_{\mathrm{s}} \rightarrow 0$ длительность солитона стремится к нулю, а амплитуда поля деформации остается конечной. Это свойство является следствием интеграла (14) и не выполняется в случае уравнения РМБ, амплитуда односолитонного решения которого обратно пропорциональна длительности.

Проведенный анализ показывает, что в рассматриваемой задаче имеет место асимметрия по полярности обеих компонент акустического импульса: компонента $\Omega_{2}$ стремится уменьшить частоту квантового перехода, а знак площади $\Omega_{1}$ определен знаком $q$. Впервые у электромагнитных ПКИ в анизотропной среде такая асимметрия была обнаружена в работе [37]. Ее проявления для системы МРМБ в частных случаях были подробно изучены в работах [28]-[30] $(d=0)$ и [31] $(q=0)$.

В пределе $\tau_{\mathrm{s}} \rightarrow \tau_{\mathrm{r}}$ интервал между горбами продольной и поперечной компонент импульса становится неограниченным. Переменные $\Omega_{1}, \Omega_{2}, U, V$ и $W$ принимают в центре импульса следующие значения: $-2 \omega_{0} q \tau_{\mathrm{r}}^{2} /\left(q^{2} \tau_{\mathrm{r}}^{4}+d^{2}\right), 2 \omega_{0}\left(q^{2} \tau_{\mathrm{r}}^{2}-d^{2}\right) /\left(q^{2} \tau_{\mathrm{r}}^{4}+d^{2}\right)$, $-2 W_{0} q \tau_{\mathrm{r}}^{2} /\left(q^{2} \tau_{\mathrm{r}}^{4}+d^{2}\right), 0$ и $W_{0}\left(q^{2}+d^{2}-1\right) \tau_{\mathrm{r}}^{2} /\left(q^{2} \tau_{\mathrm{r}}^{4}+d^{2}\right)$ (данным значениям переменных соответствуют на рис. 1 и 2 пунктирные прямые). Видно, что тогда выполняется соотношение $1+\Omega_{2} / \omega_{0}=W / W_{0}$. Это означает, что в центре импульса среда всегда находится в термодинамически равновесном состоянии: если происходит инверсия

3 Теоретическая и математическая физика, т. 151, № 2, 2007 г. 


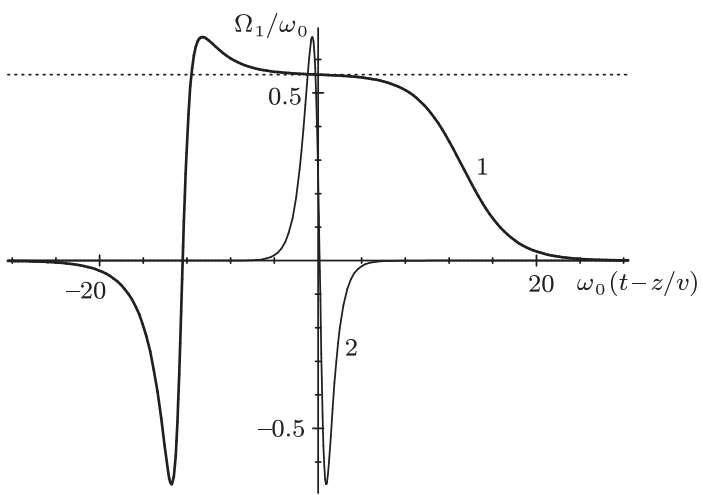

a

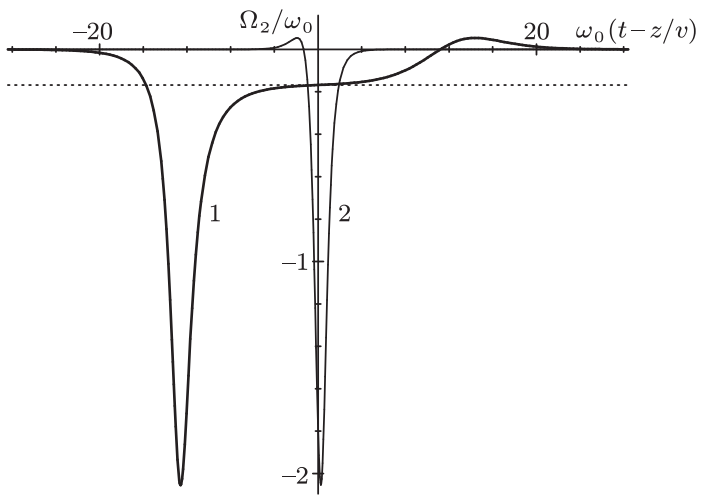

б

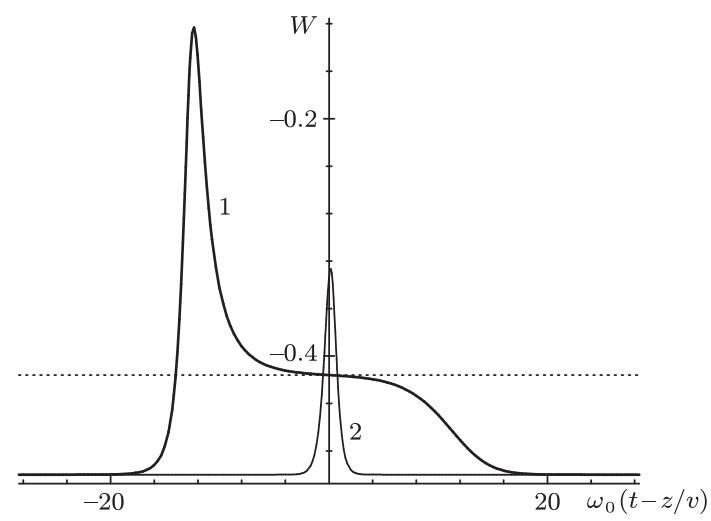

B

Рис. 2. Профили компонент поля деформации (а), (б) и инверсии населенности (в) в случае $\tau_{\mathrm{s}}<\tau_{\mathrm{r}}$ для $r q>0, \tau_{\mathrm{s}}=2.3675$ (сплошная линия 1 ) и $r q<0$, $\tau_{\mathrm{s}}=0.8$ (сплошная линия 2). Значения остальных параметров и пунктирные прямые те же, что на рис. 1. 
населенностей уровней при прохождении импульса $(W>0)$, то энергия возбужденного состояния окажется меньше энергии основного вследствие относительного смещения квантовых уровней, вызванного квадрупольным эффектом Штарка.

Перейдем теперь к рассмотрению солитонного решения уравнения МСГ (18), следуя работам [34] и [35]. Возьмем за исходное решение данного уравнения нулевой фон: $\theta=0$. В этом случае компоненты решения $\chi$ пары Лакса $(23)$ с $\varkappa=\nu$ имеют вид

$$
\chi_{1}=c_{1} e^{\zeta / 2}+c_{2} e^{-\zeta / 2}, \quad \chi_{2}=c_{1} e^{\zeta / 2}-c_{2} e^{-\zeta / 2},
$$

где

$$
\zeta=\frac{\left(T-b \tau_{\mathrm{p}}^{2} z\right)}{\tau_{\mathrm{p}}}, \quad \tau_{\mathrm{p}}=\nu^{-1} .
$$

Если параметр $\tau_{\mathrm{p}}$ веществен, то можно положить $\chi_{2}=\chi_{1}^{*}$. Тогда, подставив выражения для компонент $\chi$ в соотношение (29), получим вещественное односолитонное решение уравнения МСГ

$$
\theta=2 \arccos \frac{s-\operatorname{th} \zeta}{\sqrt{1-2 s \operatorname{th} \zeta+s^{2}}},
$$

где $s=\tau_{\mathrm{c}} / \tau_{\mathrm{p}}$. Если $f(\tau)=0$, то переменную $\zeta$ можно записать как $\zeta=\left(t-z / v_{\mathrm{M}}\right) / \tau_{\mathrm{p}}$, в котором скорость $v_{\mathrm{M}}$ солитона $(38)$ в лабораторной системе координат равна

$$
v_{\mathrm{M}}=a\left(1+a b \tau_{\mathrm{p}}^{2}\right)^{-1} .
$$

Соответствующее выражение для компоненты $\Omega_{1}$ солитона имеет вид

$$
\Omega_{1}=\frac{\operatorname{sech} \zeta}{\tau_{\mathrm{p}}} \frac{1-s \operatorname{th} \zeta}{1-2 s \operatorname{th} \zeta+s^{2}} .
$$

Компонента $\Omega_{2}$ связана с $\Omega_{1}$ соотношением (14). Выражения для продольной и поперечной компонент поля деформации импульса следуют из соотношений (8). Сопутствующая динамика спиновых состояний описывается уравнениями (17). Получающиеся при этом выражения следуют из формул (32)-(37), если в них положить $\tau_{\mathrm{s}}=\omega_{0} \tau_{\mathrm{p}}, q=0$ и учесть, что $\tau_{\mathrm{s}} \ll 1$. Последнее условие соответствует приближению СП. Требование $q=0$ связано с тем, что, как предполагалось при выводе уравнения МСГ, величины $\Omega_{2}$ и $\omega_{0}$ должны быть одного порядка (см. (15)).

"Площадь" компоненты $\Omega_{1}$ солитона $A_{\mathrm{s}}=\left.\theta\right|_{T \rightarrow \infty}$, которой можно определить его топологический заряд $A_{\mathrm{s}} / 2 \pi$, равна

$$
A_{\mathrm{s}}= \begin{cases}2 \pi \operatorname{sign}\left(\tau_{\mathrm{p}}\right), & |s|<1 \\ 0, & |s|>1\end{cases}
$$

Таким образом, сушествуют три семейства односолитонных импульсов с топологическими зарядами $1,-1$ и 0 . Первые два семейства есть также у уравнения СГ и соответствуют $2 \pi$-импульсам (кинкам и антикинкам). Солитоны из третьего семейства будем называть нейтральными кинками. В отличие от бризерного решения 
уравнения СГ, тоже имеющего нулевую “площадь" (0л-импульс), эти решения ста-

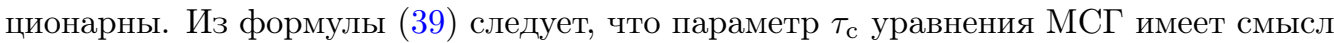
критической длительности, разделяющей $2 \pi$-импульсы $\left(\left|\tau_{\mathrm{p}}\right|>\tau_{\mathrm{c}}\right)$ и нейтральные кинки $\left(\left|\tau_{\mathrm{p}}\right|<\tau_{\mathrm{c}}\right)$.

Если $|s|<1$, то компонента $\Omega_{1}$ односолитонного импульса является однополярной и состоящей из одного горба при $|s|<1 / \sqrt{2}$ или двух симметричных горбов при $|s|>1 / \sqrt{2}$. В первом случае ее амплитуда равна $2 \sqrt{1-s^{2}} /\left|\tau_{\mathrm{p}}\right|$. Во втором случае она достигает наибольшего по абсолютной величине значения $1 / \tau_{\mathrm{c}}$, а максимумы горбов разделены временнь́м интервалом

$$
2\left|\tau_{\mathrm{p}}\right| \operatorname{arcsh} \sqrt{\frac{s^{2}}{1-s^{2}}-1} .
$$

При этом значение $\Omega_{2}$ между горбами компоненты $\Omega_{1}$ стремится к $-2 \omega_{0}$. Эта компонента асимметрична по полярности и сдвигает положения основного и возбужденных уровней за счет эффекта Штарка таким образом, что в центре импульса они меняются местами. Подобная ситуация, как было отмечено выше, имеет место и для системы МРМБ.

В случае нейтрального кинка компонента $\Omega_{1}$ состоит из двух антисимметричных горбов, временно́й промежуток между пиками которых равен

$$
2\left|\tau_{\mathrm{p}}\right| \operatorname{arcch} \sqrt{1+\frac{s^{2}}{s^{2}-1}} .
$$

Амплитудные значения компонент здесь такие же, как в случае $1 / \sqrt{2}<|s|<1$. В соответствии с предположениями, использованными при выводе уравнения МСГ, кривые 2 на рис. 2 дают представление о профиле компонент поля деформации и динамике инверсии населенности при прохождении нейтрального кинка.

При $|s|=1$ интервал между горбами становится неограниченным. Компонента $\Omega_{1}$ состоит из одного горба, амплитуда и модуль площади которого равны соответственно $1 / \tau_{\text {с и }} \pi$.

Из теории СИП известно, что прохождение солитона уравнения СГ вызывает полную инверсию населенности квантовых уровней при любом значении параметра $\tau_{\mathrm{p}}$. Если $\tau_{\mathrm{c}} \neq 0$, то, как следует из формул (17) и (38), возбуждение квантовых частиц оказывается полным только в случае $|s|<1$. Действительно, для инверсии населенности $W$ в центре импульса, где достигается ее наибольшее изменение, получаем

$$
W= \begin{cases}-W_{0}, & |s|<1, \\ W_{0}-\frac{2 W_{0}}{s^{2}}, & |s|>1\end{cases}
$$

Если $|s| \leqslant \sqrt{2}$, то суммарная населенность спиновых подуровней с $S_{z^{\prime}}= \pm 1$ будет больше, чем у подуровня с $S_{z^{\prime}}=0$. Но эти уровни смещаются за счет действия компоненты $\Omega_{2}$, в результате чего энергия уровня с $S_{z^{\prime}}=0$ в центре акустического импульса больше энергии уровней с $S_{z^{\prime}}= \pm 1$. Мы видим, что динамика эффективных спинов в случае уравнения МСГ такая же, как у системы МРМБ. 
Итерации преобразований (24)-(27) и (28), (29) на нулевом фоне дают возможность построить многосолитонные решения уравнений МРМБ и МСГ. Эти решения описывают взаимодействие солитонов, рассмотренных в этом разделе, и бризеров, данные рассеяния которых содержат пары комплексно-сопряженных собственных значений дискретного спектра. Нетрудно показать, что результатом их столкновений будут сдвиги, величина которых такая же, как у солитонных и бризерных решений уравнений РМБ и СГ. Однако, как было обнаружено в работе [35] для уравнения МСГ, ход взаимодействия солитонов существенным образом зависит от соотношений между их свободными параметрами и коэффициентами уравнений. Свойства бризерных решений и особенности столкновений солитонов уравнений МРМБ и МСГ мы предполагаем обсудить в отдельной статье.

В завершение этого раздела коснемся кратко эволюции произвольных быстроубывающих начальных профилей компонент $\Omega_{1}$ и $\Omega_{2}$. Так как рассмотренные здесь уравнения калибровочно-эквивалентны хорошо изученным в рамках МОЗР уравнениям, то присутствуют общие черты: начальное условие распадается при $t \rightarrow \infty$ на солитонную часть и диспергирующий шлейф, связанный с непрерывной частью данных рассеяния. Новой особеностью является то, что из начального профиля компоненты $\Omega_{2}$ выделится недиспергирующий стационарный импульс, форма которого полностью определена функцией $f(\tau)$ в интеграле (14). Форма солитонов асимптотически стремится к их форме в случае $f(\tau)=0$. Это, в частности, означает, что амплитуда солитонов ограничена.

\section{6. ЗАКЛЮЧЕНИЕ}

В настоящей работе показано, что динамика продольно-поперечного акустического импульса в деформированном парамагнитном кристалле описывается интегрируемой в рамках МОЗР системой МРМБ (7), (12), (13), а также интегрируемым уравнением МСГ (18), если длительность импульса удовлетворяет условию спектрального перекрытия (16). Анализ солитонных решений этих уравнений выявил качественно новые особенности в поведении эффективных спинов и компонент поля деформации импульса по сравнению со случаями сугубо поперечных или продольных акустических полей. Оказалось, что характер взаимодействия импульса и среды существенно зависит от соотношений между свободными параметрами солитонов и коэффициентами уравнений. По этой причине уравнение МСГ, например, имеет новый тип решений - нейтральные кинки, топологический заряд которых равен нулю. Особенностью системы МРМБ является то, что она обладает несингулярными рационально убывающими солитонными решениями.

По нашему мнению, уравнения МРМБ и МСГ, как и другие уравнения, калибровочно-эквивалентные хорошо известным интегрируемым уравнениям (см., например, [38]), заслуживают отдельного изучения и специального развития для них аппарата МОЗР и методов теории солитонов. Так, представляют интерес задачи квантования нейтральных кинков, гамильтоновой формулировки уравнений и описания данных рассеяния рационально убывающих солитонов. Изучение автомодельных 
редукций может способствовать появлению новых подходов в теории теста Пенлеве. Кроме того, физическая сторона исследования калибровочно-эквивалентных уравнений открывает широкое поле деятельности. Так, интегрируемая модель, описывающая распространение циркулярно поляризованных электромагнитных ПКИ в анизотропной среде и обобщающая уравнения РМБ, была выведена в работе [39]. Модифицированная версия этой модели, подобная изученным в данной статье, может также найти приложения в нелинейной оптике и физической акустике.

Благодарности. Работа выполнена при финансовой поддержке РФФИ (грант № 05-02-16422).

\section{Список литературы}

[1] S. L. McCall, E. L. Hahn, Phys. Rev. Lett., 18 (1967), 908.

[2] G. L. Lamb, Rev. Mod. Phys., 43 (1971), 99.

[3] J.D. Gibbon, J. C. Eilbeck, J. Phys. A, 5 (1972), L122; P. J. Caudrey, J. D. Gibbon, J. C. Eilbeck, R. K. Bullough, Phys. Rev. Lett., 30 (1973), 237; J. Phys. A, 6 (1973), L53; G. L. Lamb, Phys. Rev. Lett., 31 (1973), 196.

[4] В.Е. Захаров, С. В. Манаков, С. П. Новиков, Л. П. Питаевский, Теория солитонов. Метод обратной задачи, Наука, М., 1980; Дж. Л. Лэм, Введение в теорию солитонов, Мир, М., 1983.

[5] Л. А. Тахтаджян, Л. Д. Фаддеев, Гамильтонов подход в теории солитонов, Наука, М., 1986.

[6] В.Е. Захаров, А.Б. Шабат, ЖЭЭТФ, 61 (1971), 118.

[7] Г. Агарвал, Нелинейная волоконная оптика, Мир, М., 1996.

[8] J. C. Eilbeck, J. Phys. A, 5 (1972), 1355.

[9] J. C. Eilbeck, J. D. Gibbon, P. J. Caudrey, R. K. Bullough, J. Phys. A, 6 (1973), 1337; J. D. Gibbon, P. J. Caudrey, R. K. Bullough, J. C. Eilbeck, Lett. Nuovo Cimento, 8 (1973), 775 .

[10] N. S. Shiren, Phys. Rev. B, 2 (1970), 2471.

[11] Г. А. Денисенко, ЖЭТФ, 60 (1971), 2269.

[12] В. В. Самарцев, Б. П. Смоляков, Р. З. Шарипов, Писъма в ЖКЭТФ, 20 (1974), 644.

[13] С.А. Ахманов, В.А. Выслоух, А.С. Чиркин, Оптика фемтосекундных лазерных импульсов, Наука, М., 1988; В.Э. Гусев, А.А. Карабутов, Лазерная оптоакустика, Наука, М., 1991; С.А. Ахманов, В.Э. Гусев, УФН, 162:3 (1992), 3; http://ufn.ioc.ac.ru/archive/russian/abstracts/abst1338.html.

[14] Э. М. Беленов, А.В. Назаркин, Писъма в ЖЖЭТФ, 51 (1990), 252; Э. М. Беленов, А. В. Назаркин, В. А. Ущаповский, ЖЭТФ, 100 (1991), 762.

[15] A. Kujawski, Z. Phys. B, 85 (1991), 129.

[16] S. V. Sazonov, E. V. Trifonov, J. Phys. B, 27 (1994), L7.

[17] S. V. Sazonov, J. Phys.: Condens. Matter, 4 (1992), 6485; 6 (1994), 6295; C. В. Сазонов, KЭТ $\Phi, 118$ (2000), 20.

[18] С. А. Альтшулер, Б. М. Козырев, Электронный парамагнитный резонанс соединений элементов промежуточных групп, Наука, М., 1972.

[19] Дж. Такер, Р. Рэмптон, Гиперзвук в физике твердого тела, Мир, М., 1975.

[20] M. Agrotis, N. M. Ercolani, S. A. Glasgow, J. V. Moloney, Physica D, 138 (2000), 134.

[21] С. В. Воронков, С. В. Сазонов, ЖКЭТФ, 120 (2001), 269; А. А. Заболотский, ЖЖЭТФ, 123 (2003), 560; С. В. Сазонов, ЖКЭТФ, 124 (2003), 803.

[22] А.А. Заболотский, ЖЭТФ, 123 (2003), 1239.

[23] С. В. Сазонов, Н. В. Устинов, ЖЭТФ, 127 (2005), 289. 
[24] S. V. Sazonov, N. V. Ustinov, Phys. Rev. E, 73 (2006), 056614; nlin.PS/0603040.

[25] E. H. Jacobsen, K. W. H. Stevens, Phys. Rev., 129 (1963), 2036.

[26] В. А. Красильников, В. В. Крылов, Введение в физическую акустику, Наука, М., 1984.

[27] Ч. Киттель, Введение в физику твердого тела, Физматлит, М., 1963.

[28] С. О. Елютин, ЖЭТФ, 128 (2005), 17.

[29] С. В. Сазонов, Н. В. Устинов, Квант. электроника, 35 (2005), 701.

[30] С. В. Сазонов, Н. В. Устинов, ЖЭТФ, 129 (2006), 849.

[31] N. V. Bakhar, N. V. Ustinov, Proc. SPIE, 6181 (2006), 61810Q; nlin.SI/0512068.

[32] M. D. Kruskal, "The Korteweg-de Vries equation and related evolution equations", Nonlinear Wave Motion, Proc. AMS-SIAM Summer Sem. (Potsdam, NY, 1972), Lect. Appl. Math., 15, Amer. Math. Soc., Providence, RI, 1974, 61; H.-H. Chen, Phys. Rev. Lett., 33 (1974), 925.

[33] A. Nakamura, J. Phys. Soc. Japan, 49 (1980), 1167; А. Б. Борисов, С. А. Зыков, ТМФ, 115:2 (1998), 199.

[34] С. В. Сазонов, Н. В. Устинов, Писъма в ЖЭЭФ, 83 (2006), 573.

[35] С. В. Сазонов, Н. В. Устинов, ЖЭТФ, 130 (2006), 646.

[36] V. B. Matveev, M. A. Salle, Darboux Transformations and Solitons, Springer Ser. Nonlinear Dynam., Springer, Berlin-Heidelberg, 1991.

[37] А.И. Маймистов, Дж.-Г. Капуто, Оптика и спектроскопия, 94 (2003), 275.

[38] А.Б. Борисов, С. А. Зыков, М. В. Павлов, ТМФ, 131:1 (2002), 126.

[39] А.А. Заболотский, ЖЭТФ, 125 (2004), 1229. 